\title{
Numerical Simulation of the fluid-structure interaction between air blast waves and free-standing plates
}

\author{
Nayden Kambouchev ${ }^{\mathrm{a}}$, Ludovic Noels ${ }^{\mathrm{a}, \mathrm{b}, 1}$, Raul Radovitzky ${ }^{\mathrm{a}, *}$ \\ ${ }^{a}$ Department of Aeronautics and Astronautics, \\ Massachusetts Institute of Technology \\ Cambridge, MA 02139 \\ ${ }^{\mathrm{b}}$ LTAS-Milieux Continus $\& 3$ Thermomécanique \\ University of Liège, Chemin des Chevreuils 1, B-4000 Liège, Belgium
}

\begin{abstract}
A numerical method is used to compute the flow field corresponding to blast waves of different incident profiles propagating in air and impinging on free-standing plates. The method is suitable for the consideration of compressibility effects in the fluid and their influence on the plate dynamics. The history of the pressure experienced by the plate is extracted from numerical simulations for arbitrary blast strengths and plate masses and used to infer the impulse per unit area transmitted to the plate. The numerical results complement some recent analytical solutions in the intermediate range of plate masses and arbitrary blast intensities where exact solutions are not available. The resulting beneficial effect of the fluid-structure interaction (FSI) in reducing transmitted impulse in the presence of compressibility effects is discussed. In particular, it is shown that in order to take advantage of the impulse reduction provided by the FSI effect, large plate displacements are required which, in effect,
\end{abstract}


may limit the practical applicability of exploiting FSI effects in the design of blastmitigating systems.

Key words: Air blast waves, Fluid-structure interaction, compressibility effects

\section{Introduction}

Recently there has been significant interest in understanding the influence of fluid-structure interaction (FSI) on the blast loading of structures. It was shown in the pioneering work of G.I. Taylor, [1], that FSI reduces the amount of impulse transmitted to free-standing plates and that this effect is more pronounced for lighter plates. The reduction of transmitted impulse is due to the pressure relief experienced by the structure caused by its motion. Taylor's analysis is restricted to the linear case of incident blast waves of exponential profile in which the pressure wave does not cause any significant changes in the fluid density. This assumption is applicable in the case of underwater explosions, as the pressure level required for water to undergo non-negligible compressibility effects is in the order of $100 \mathrm{kbar}$, which exceeds conventional situations. The beneficial influence of FSI in potentially mitigating the effect of blast has recently been explored as a basis for the design of sandwich structures with increased blast resistance, [2-10].

Although the compressible case is not amenable to analytic treatment for the whole range of plate masses and blast intensities regardless of the blast wave

\footnotetext{
* Corresponding author. Tel: +1-617-252-1518, Fax: +1-617-253-0361, E-mail: rapa@mit.edu

1 Postdoctoral scholar at the Belgian National Fund for Scientific Research (FNRS)
} 
profile, the authors have recently derived some results in the asymptotic limits of very heavy and very light plates for the cases of uniform [11] and exponential [12] incident blast profiles. It is important to emphasize that although the uniform profile has some interest due to its analytic tractability and the exponential profile because it presumably provides a reasonable approximation to blast waves $[13,14]$, neither profile does actually correspond to the exact solution of a blast wave caused by a point explosion, which was derived in closed (implicit) form by J. von Neumann [15]. Although the simplified exponential pressure profile is attractive due to its simplicity, it is not suitable for initializing the flow in numerical simulations, as it is not clear what the corresponding density and velocity fields consistent with the governing differential equations of compressible flow are.

In this paper, we use a numerical method to conduct simulations of the interaction between blast waves of different profiles and intensities with free-standing plates of varying mass. The method is based on a Lagrangian formulation of the equations of compressible flow. In the Lagrangian framework, the dynamic response of the plate and its interaction with the flow can be simply modeled by modifying the governing equations and adding the plate's mass at the material location of the plate. Following the classic approach of von Neumann and Richtmyer [16], a shock capturing scheme based on artificial viscosity is adopted. The numerical method is verified by comparisons with exact solutions in the acoustic and nonlinear compressible range in the asymptotic limits where exact solutions have been derived [12,11]. Special attention is then paid to the computation of blast-structure interaction in the intermediate asymptotic range of plate masses where exact solutions are unavailable. The analysis includes the cases of uniform, exponential and planar explosion 
(von Neumann-Sedov) profiles. The analysis of the role of the structure supports has been omitted purposely, in an attempt to isolate and highlight the role of FSI on impulse transmission to structures, as was originally done by Taylor but in the case of a compressible fluid. Clearly, the role of the supports is to reduce the momentum acquired by the structure, at the expense of creating possible large reaction forces.

The numerical calculations play an important role in complementing recent analytical work $[12,11]$ by providing the necessary values of transmitted impulse which, in combination with the asymptotic results, are used for the development of practical formula encompassing the whole range of behavior.

This paper is organized as follows: In Section 2, the continuum problem is formulated and the numerical method employed is described. Section 3 is devoted to the presentation of numerical results including verification cases and applications. The paper concludes in Section 4 with a summary of findings and conclusions.

\section{Problem Statement and Numerical Approach}

[Fig. 1 about here.]

The problem of interest concerns the interaction of a shock wave traveling in compressible medium with a free-standing plate of thickness $h_{p}$ and density $\rho_{p}$ (Figure 1). The plate is positioned initially at $x=0$ while the fluid medium on its left side is assumed to be an ideal calorically-perfect gas at rest with density $\rho_{0}$ and pressure $p_{0}$. A constant pressure $p_{0}$ is applied on the right (free) side of the plate at all times in order to balance the loading until the arrival 
of the wave. Three different wave shapes are considered: uniform, exponential and planar explosion. The quantities of interest are the pressure histories on the plate's surface, the displacement, velocity and impulse of the plate.

In order to facilitate the description of the dynamics of the plate a Lagrangian formulation for the fluid motion is adopted. In the Lagrangian framework, the continuum equations governing the problem are:

- The kinematic relations for the material velocity and acceleration:

$$
\begin{aligned}
V & =\frac{\partial x}{\partial t} \text { and } \\
A & =\frac{\partial V}{\partial t},
\end{aligned}
$$

where the Eulerian coordinate $x$, the velocity $V$ and acceleration $A$ of a material particle are functions of the Lagrangian coordinate $X$ and the time $t$.

- The momentum conservation equation:

$$
\rho_{0} A=-\frac{\partial p}{\partial X}
$$

where $\rho_{0}$ is the initial density of the particle with Lagrangian coordinate $X$ and $p$ is the pressure.

- The equation of state, which is modified to include a viscous dissipation term $Q$ :

$$
p=\rho R T-Q=(\gamma-1) \rho_{0} \frac{e}{F}-Q,
$$

where $R$ is the ideal gas constant, $T$ is the absolute temperature, $\gamma=\frac{C_{p}}{C_{v}}$ is the specific heat ratio, where $C_{p}$ and $C_{v}$ are the specific heats at constant pressure and volume, $e=C_{v} T$ is the internal energy and $F=\frac{\partial x}{\partial X}$ is the deformation gradient. The viscous dissipation term is required for stabilization of the numerical scheme and consists of the original quadratic term of 
von Neumann and Richtmyer [16] and a linear term due to Kuropatenko $[17]:$

$$
Q=\left\{\begin{array}{ll}
-\rho_{0}\left(K_{1} D \Delta\right)^{2}-\rho_{0} a_{0} K_{2}|D| \Delta, & D<0 \\
0, & D \geq 0
\end{array},\right.
$$

where $D=\frac{1}{F} \frac{\partial F}{\partial t}$ is the deformation rate, $K_{1}$ and $K_{2}$ are artificial viscosity coefficients, $a=\sqrt{\gamma(\gamma-1) e}$ is the local speed of sound and $\Delta$ is the width of the smeared shock which must be of the order of the grid spacing for numerical stability.

- The energy conservation equation, including the viscous dissipation term:

$$
\frac{\partial e}{\partial t}=\left[(1-\gamma) e+\frac{Q}{\rho}\right] D
$$

where $\rho$ is the current density of the particle.

Following Taylor [1], the plate is treated as a rigid body on the grounds that the time scales of the elastic wave propagation within the plate are several orders of magnitude smaller than the time scale of the fluid structure interaction. With this assumption, the motion of the plate is described by Newton's second law:

$$
\frac{d^{2} \xi}{d t^{2}}=\frac{p_{p}}{m_{p}}
$$

where $\xi$ is the location of the plate, $m_{p}=\rho_{p} h_{p}$ is the mass of the plate and $p_{p}$ is the overpressure acting on it. The initial conditions for the plate position $\xi$ are $\xi(t=0)=0$ and $\frac{d \xi}{d t}(t=0)=0$, while the initial conditions for the gas particles are $x(X, t=0)=X$ and $V(X, t=0)=0$.

The numerical discretization is based on the original finite difference method proposed by von Neumann and Richtmyer [16]. Following closely the algorithmic approach in [18], we discretize the domain of interest into $N+1$ equally 
spaced points. The coordinates of these points are given by $x_{0}^{(n)}=X^{(n)}=$ $(n-N) \Delta X$ where $n=1,2, \ldots, N$ is the point number and $\Delta X$ is the grid spacing. Time is discretized into variable time steps $\Delta t_{j}, j=1,2, \ldots$ and the discretized solution is obtained at times $t_{0}=0, t_{1}, \ldots, t_{j-1}, t_{j}=t_{j-1}+\Delta t_{j}, \ldots$ The final difference approximation for the particle velocity leads to:

$$
x_{j+1}^{(n)}=x_{j}^{(n)}+\Delta t_{j+1} V_{j+\frac{1}{2}}^{(n)}
$$

where the velocity $V$ is computed in the middle of the time intervals, while the finite difference approximation for the acceleration gives:

$$
V_{j+\frac{1}{2}}^{(n)}=V_{j-\frac{1}{2}}^{(n)}+\frac{1}{2}\left(t_{j}+t_{j+1}\right) A_{j}^{(n)}
$$

The acceleration needed for the previous equation is obtained from the discretized momentum conservation equation:

$$
A_{j}^{(n)}=-\frac{1}{\rho_{0}^{(n)}} \frac{p_{j}^{\left(n+\frac{1}{2}\right)}-p_{j}^{\left(n-\frac{1}{2}\right)}}{\Delta X} .
$$

The remaining discretized equations are: 


$$
\begin{aligned}
p_{j+1}^{\left(n+\frac{1}{2}\right)}= & (\gamma-1) \rho_{0} \frac{e_{j+1}^{\left(n+\frac{1}{2}\right)}}{F_{j+1}^{\left(n+\frac{1}{2}\right)}}-Q_{j+\frac{1}{2}}^{\left(n+\frac{1}{2}\right)}, \\
Q_{j+\frac{1}{2}}^{\left(n+\frac{1}{2}\right)} & =-\rho_{0}\left(K_{1} \Delta x_{j+\frac{1}{2}}^{\left(n+\frac{1}{2}\right)} D_{j+\frac{1}{2}}^{\left(n+\frac{1}{2}\right)}\right)^{2}- \\
& \rho_{0} K_{2} a_{j}^{\left(n+\frac{1}{2}\right)} \Delta x_{j+\frac{1}{2}}^{\left(n+\frac{1}{2}\right)}\left|D_{j+\frac{1}{2}}^{\left(n+\frac{1}{2}\right)}\right|, \\
a_{j}^{\left(n+\frac{1}{2}\right)}= & \sqrt{\gamma(\gamma-1) e_{j}^{\left(n+\frac{1}{2}\right)}}, \\
D_{j+\frac{1}{2}}^{\left(n+\frac{1}{2}\right)}= & \frac{2}{\Delta t_{j+1}} \frac{F_{j+1}^{\left(n+\frac{1}{2}\right)}-F_{j}^{\left(n+\frac{1}{2}\right)}}{F_{j+1}^{\left(n+\frac{1}{2}\right)}+F_{j}^{\left(n+\frac{1}{2}\right)}}, \\
\Delta x_{j+\frac{1}{2}}^{\left(n+\frac{1}{2}\right)}= & \frac{1}{2}\left(x_{j+1}^{(n+1)}-x_{j+1}^{(n)}+x_{j}^{(n+1)}-x_{j}^{(n)}\right), \\
e_{j+1}^{\left(n+\frac{1}{2}\right)}= & \frac{e_{j}^{\left(n+\frac{1}{2}\right)}+\left(\frac{1-\gamma}{2} e_{j}^{\left(n+\frac{1}{2}\right)}+\frac{Q_{j+\frac{1}{2}}^{\left(n+\frac{1}{2}\right)}}{\rho_{j+\frac{1}{2}}^{\left(n+\frac{1}{2}\right)}}\right) \Delta t_{j+1} D_{j+\frac{1}{2}}^{\left(n+\frac{1}{2}\right)}}{2+\frac{\gamma-1}{2} \Delta t_{j+1} D_{j+\frac{1}{2}}^{\left(n+\frac{1}{2}\right)}} \\
\rho_{j+\frac{1}{2}}^{\left(n+\frac{1}{2}\right)}= & \frac{\rho_{0}^{\left(n+\frac{1}{2}\right)}}{2}\left(\frac{1}{F_{j+1}^{\left(n+\frac{1}{2}\right)}}+\frac{1}{F_{j}^{\left(n+\frac{1}{2}\right)}}\right), \\
\rho_{0}^{\left(n+\frac{1}{2}\right)}= & \frac{\rho_{0}^{(n+1)}+\rho_{0}^{(n)}}{2},
\end{aligned}
$$

where the discrete deformation gradient $F$ is given by

$$
F_{j}^{\left(n+\frac{1}{2}\right)}=\frac{x_{j}^{(n+1)}-x_{j}^{(n)}}{\Delta X} .
$$

The solution process consists of applying the following sequence of steps to each node $j$ in the fluid domain $j=0, \cdots, N-1$ ) except the last one $j=N$ which is treated separately. The first step in each time step iteration is to compute the stable time step size:

$$
\Delta t_{j}=\alpha \min _{n=0,1, \ldots, N-1}\left(\frac{x_{j-1}^{(n)}-x_{j-1}^{(n-1)}}{a_{j-1}^{(n-1)}}\right),
$$

where $0 \leq \alpha \leq 1$ is an appropriately chosen time factor. This step is followed by the computation of the deformation gradient $F_{j}^{\left(n+\frac{1}{2}\right)}(19)$, the deformation rate $D_{j+\frac{1}{2}}^{\left(n+\frac{1}{2}\right)}(14)$ and the Eulerian spacing $\Delta x_{j+\frac{1}{2}}^{\left(n+\frac{1}{2}\right)}(15)$. From these the viscous 
dissipation $Q_{j+\frac{1}{2}}^{\left(n+\frac{1}{2}\right)}(12)$ and the internal energy $e_{j+1}^{\left(n+\frac{1}{2}\right)}(16)$ can be computed. At the next step the pressure $p_{j+1}^{\left(n+\frac{1}{2}\right)}$ is obtained from (11) and substituted in the expression of the acceleration $A_{j}^{(n)}(10)$. A straightforward substitution into the equations for the material velocity $V_{j+\frac{1}{2}}^{(n)}(9)$ and the particle location $x_{j+1}^{(n)}$ (8) completes the cycle for the time step. The motion of node $N$ corresponding to the plate is computed using a forward Euler scheme:

$$
\begin{aligned}
\xi_{j+1} & =\xi_{j}+\Delta t_{j+1}\left(\frac{d \xi}{d t}\right)_{j} \\
\left(\frac{d \xi}{d t}\right)_{j+1} & =\left(\frac{d \xi}{d t}\right)_{j}+\Delta t_{j+1}\left(\frac{d^{2} \xi}{d t^{2}}\right)_{j} \\
\left(\frac{d^{2} \xi}{d t^{2}}\right)_{j} & =\frac{p_{j}^{\left(N-\frac{1}{2}\right)}}{m_{p}}
\end{aligned}
$$

where the pressure acting on the plate is approximated by the pressure computed in the mid-position of the last fluid element. The boundary condition on the left end depends on the particular problem and will be discussed opportunely. Initial conditions are also problem dependent but in all cases include the condition that material and spatial coordinates coincide at $t=0$ : $x(X, t=0)=X$.

\section{Numerical Results}

In this section the numerical method is verified against known exact solutions for uniform shocks reflecting on fixed boundaries and then used for simulating the interaction of blast waves of different incident profiles and intensities with plates of varying mass. In all cases, the time profiles of pressure experienced by the plate and the transmitted impulse $I_{p}$ are extracted from the simulations and analyzed in order to reveal the influence of FSI. The gas properties for air 
are assumed throughout, i.e. specific heat ratio $\gamma=1.4$, initial density $\rho_{0}=$ $1.225 \mathrm{~kg} / \mathrm{m}^{3}$ and initial temperature $T_{0}=298 \mathrm{~K}$, which give $p_{0}=1.048 \mathrm{bar}=$ $1 \mathrm{~atm}$ and a reference sound speed $a_{0}=346 \mathrm{~m} / \mathrm{s}$.

\subsection{Verification of the numerical method: Normal reflection of uniform pla- nar shocks on a fixed boundary}

[Fig. 2 about here.]

The case of the normal reflection of uniform shocks on a fixed boundary provides a good basis for verification of the numerical method. One of the basic implications of gas compressibility is the nonlinear dependence of the pressure reflected from a fixed rigid wall on the magnitude of the incident shock pressure. In the case of air, the reflected pressure is [19]:

$$
p_{r}=2 p_{s} \frac{7 p_{0}+4 p_{s}}{7 p_{0}+p_{s}}
$$

where $p_{s}$ and $p_{r}$ are the incident and reflected pressures, respectively. A pressure reflection coefficient $C_{R}$ may be defined as:

$$
C_{R}=\frac{p_{r}}{p_{s}}=2 \frac{7 p_{0}+4 p_{s}}{7 p_{0}+p_{s}}
$$

It is clear from equations (24) and (25) that the reflected pressure ranges from a minimum value of twice the incident pressure, which corresponds to the acoustic range, to a maximum value of eight times the incident pressure, which is the limit of increasingly strong shocks for which the ideal gas equation of state with constant $\gamma$ is valid:

$$
2 \leq C_{R} \leq 8
$$


In order to generate a uniform shock, a constant piston velocity $V_{L}(X=0, t \geq$ $0)=u_{s}$, where $u_{s}$ is the velocity of the particles behind the shock, is applied as boundary condition at the left end of the fluid domain, i.e. $v_{j+\frac{1}{2}}^{(0)}=u_{s}\left(p_{s}\right)$ for all time steps $j=0, \cdots$. For a desired shock overpressure, $u_{s}$ is computed from the expression:

$$
u_{s}=a_{0} \frac{5}{7} \frac{p_{s}}{p_{0}} \frac{1}{\sqrt{6 \frac{p_{s}}{p_{0}}+1}}
$$

where $a_{0}$ is the reference speed of sound of air. The domain size is $8 \mathrm{~m}$ discretized with $N=5000$ grid points and the grid node corresponding to the plate has been fixed.

The results obtained are shown in Figure 2, where the values of the reflected pressures obtained numerically have been normalized with the incident shock pressures $p_{s}$ and compared with the exact values of the reflected coefficients given by equation (25). As it can be observed in the plot, the numerical results are in agreement with the Rankine-Hugoniot theory for a wide range of shock intensities.

\subsection{Reflection of uniform shocks on free-standing plates}

In this section, we consider the FSI problem of an incident uniform shock of arbitrary intensity reflecting on a free-standing plate. The simulations are set up in the same way as in the previous example except that the boundary condition on the right end of the domain is removed and the motion of the end grid node corresponds to the dynamics of the plate, as described in Section 2.

This example provides a good basis for numerical verification of the theory presented in [11] which presents exact solutions for the asymptotic limits of 
very heavy and very light plates interacting with shocks of arbitrary intensities and for arbitrary plate masses interacting with acoustic waves.

[Fig. 3 about here.]

The derivation of the asymptotic behavior of the impulse transmission coefficient for light plates derived in [11] is based on the assumption that the maximum velocity is reached instantaneously at time $t=0^{+}$. The validity of this assumption may be assessed by conducting numerical simulations and extracting from them overpressure profiles recorded on the plate surface (point $N$ ) for different values of $\beta_{s}$. The profiles obtained for overpressure $p_{s} / p_{0}=4.49$ are shown in Figure 3. The dependence on the mass of the plate is given in terms of the compressible non-dimensional parameter proposed in the same reference:

$$
\beta_{s}=\frac{\rho_{s} U_{s} t_{i}}{\rho_{p} h_{p}}
$$

where $\rho_{s}$ is the gas density behind the shock, $U_{s}$ the shock speed, $t_{i}$ the duration of the shock pulse considered and $\rho_{p}$ and $h_{p}$ the plate density and thickness, respectively. The duration of the uniform shock $t_{i}$ is defined as the time elapsed since the shock reached the plate. It may be observed in this figure that for all values of $\beta_{s}$, the initial overpressure is the same $p_{p}(t=0)=p_{r}$. However, for increasing $\beta_{s}$, the overpressure profiles decay faster with time. In the limit $\beta_{s} \rightarrow \infty$, the overpressure drops to zero instantly after shock impact, in which case the pressure profile corresponds to a generalized delta function multiplied by a constant. As shown in [11], integration of equation (7) results in a constant plate velocity which is independent of the plate mass and achieved instantaneously, which validates the assumption in [11]. This is confirmed by the plate velocities obtained for large values of $\beta_{s}=6730,673$ 
and 67.3 which are $u_{p}=1005.5 \mathrm{~m} / \mathrm{s}, 1005.6 \mathrm{~m} / \mathrm{s}$ and $1006.5 \mathrm{~m} / \mathrm{s}$, respectively.

[Fig. 4 about here.]

The independence of the plate velocity from its mass in the case of light plates has an immediate consequence on the impulse transmission: it becomes directly proportional to the mass of the plate, $I_{p} \propto m_{p}$, and is lower for lighter plates, in direct contrast with the case of the heavy plate limit in which the impulse is independent of the mass. This can be observed in Figure 4 where the time histories of the transmitted impulse are shown. For heavy plates (small $\beta_{s}$ ) the evolution of the linear momentum of the plate with time has almost a constant slope, which corresponds to a constant force acting on the plate. On the contrary, for light plates (large $\beta_{s}$ ) the force acting on the plate decreases with time giving lower values of the transmitted impulse.

[Fig. 5 about here.]

Figure 5 summarizes and compares normalized transmitted impulse obtained numerically for a wide range of plate masses and shock intensities against the results of the theory presented in [11]. The figure shows a plot of the normalized transmitted impulse $\frac{I_{p}}{C_{R} I_{i}}$ vs. the combination of parameters $\frac{\beta_{s}}{f_{R}}$ where $I_{i}=p_{s} t_{i}$ is the impulse carried by the shock wave through the point $x=0$ were the plate not there and $f_{R}$ is defined in equation (30). The analytical solution of the transmitted momentum was developed in [11]:

$$
\frac{I_{p}}{C_{R} I_{i}}=\frac{1-e^{-\beta_{s} / f_{R}}}{\beta_{s} / f_{R}}
$$

The parameter

$$
f_{R}=\left(6 \frac{p_{s}}{p_{0}}+7\right) \sqrt{\frac{\left(6+C_{R}\right) \frac{p_{s}}{p_{0}}+7}{\left(\frac{p_{s}}{p_{0}}+7\right)\left(\left(1+6 C_{R}\right) \frac{p_{s}}{p_{0}}+7\right)\left(C_{R} \frac{p_{s}}{p_{0}}+7\right)}}
$$


characterizes the compressibility effect occurring during the wave expansion associated with the motion of a light plate acting like a free surface $[12,11]$. As it can be seen in Figure 5, an excellent agreement is found between the numerical results and the theory. This verifies the accuracy of the numerical method in the asymptotic limits of heavy and light plates for arbitrary shock intensity as well as the acoustic solution for arbitrary plate weights. In particular, for $\beta_{s} \rightarrow 0$ the curve becomes horizontal supporting the correctness of the assumption that heavy plates behave as fixed walls and therefore absorb the same impulse independently of the plate mass. For $\beta_{s} \rightarrow \infty$ the curve has slope -1 which is consistent with the assumption that all plates acquire the same maximum velocity (specifically $I_{p} / I_{i} \propto m_{p}$ while $\beta_{s} \propto 1 / m_{p}$, so that $\left.I_{p} / I_{i} \propto 1 / \beta_{s}\right)$. In addition and most importantly, the numerical results complement the exact theory, support the predictions of the empirical formula in the intermediate asymptotic range, and confirm the collapse of the impulse onto a single curve when normalized in terms of the parameter $\frac{\beta_{s}}{f_{R}}$.

[Fig. 6 about here.]

The reduction of the impulse transmitted from blast wave to light structures has been used as a basis for the design of sandwich panels with increased resistance to underwater explosions, [2-10]. It should be noted that impulse reduction is achieved owing to the motion of the plate away from the shock wave. However, in the case of air explosions, the displacement $\xi$ can become extremely large for light plates. The time evolution of $\xi$ for different values of $\beta_{s}$ is shown in Figure 6 for the somewhat mild overpressure of $p_{s} / p_{0}=4.49$. The lightest plate shown in the figure moves approximately $0.8 \mathrm{~m}$ in $t_{i}=0.001$ s, which might be a significant distance for some applications. Any obstacle to the motion of the plate will increase the transmitted impulse considerably. 


\subsection{Exponential blast profiles}

The case of exponential profiles is of interest as they are commonly adopted to approximate realistic blast waves $[13,14]$. However, this approximation of the incident loading does not provide a consistent means of initializing the gas flow, which leads to some numerical problems, especially for high-intensity blast waves. This case was discussed in analytical and numerical detail in [12]. For completeness, we summarize the results in this section.

The simulations are initialized by imposing an exponential spatial pressure distribution with a peak pressure $p_{i}$ and decay distance $d_{i}$. The initial velocity is defined from relation (27), whereas the initial density is defined from the Rankine-Hugoniot relation

$$
\rho_{s}=\rho_{0} \frac{7 p_{0}+6 p_{s}}{7 p_{0}+p_{s}}
$$

At the initial location of the blast front, the pressure is ramped linearly from $p_{0}$ to $p_{i}$ across 10 grid points in order to minimize the initial viscous overheating. The domain size and stand-off distance $d$ are chosen so that undesirable interference of waves reflecting from the left boundary and the blast-wave interaction is avoided. In combination with the stand-off distance, the initial blast peak pressure $p_{i}$ and $d_{i}$ determine the peak pressure and decay time at the point of impact on the plate $p_{s}$ and $t_{i}$, respectively.

Simulations of a blast wave interacting with a free-standing plate were conducted for different blast intensities and plate masses for the purpose of computing the transmitted impulse. Figure 7 shows a summary of the results presented in [12] and a comparison with the empirical formula giving the normalized transmitted impulse as a function of the blast intensity and normalized 
plate mass proposed in this reference:

$$
\frac{I_{p}}{I_{i}}=\gamma_{R}\left(\frac{C_{R} f_{R}}{\gamma_{R}}\right)^{\frac{\beta_{s}}{1+\beta_{s}}} \beta_{s}^{\frac{\beta_{s}}{1-\beta_{s}}}
$$

[Fig. 7 about here.]

As it may be observed in this figure, the numerical results accurately match their theoretical counterparts. However, for higher intensities, the numerical simulations suffer from spurious wave reflections stemming from the initial conditions which do not correspond to the actual solution of a blast wave propagating in air.

\subsection{Exact blast profiles corresponding to Point Explosion}

Real explosions consist of a fast localized release of a large amount of energy and/or mass usually in the form of a gas at very high temperature. A useful idealization of such process is a point energy release for which exact solutions

of the equations of fluid motion have been derived by von Neumann [15] and Sedov [20]. The solutions presented in these references only apply to the case of very intense air blasts, as long as the peak pressure of the generated blast wave remains above 10 atm [21]. The case of low intensity blast waves has been treated by Bach et al $[22,23]$. The von Neumann-Sedov solution in the particular case of one-dimensional flow is given implicitly in terms of a parameter $0 \leq \theta \leq 1$ as: 


$$
\begin{aligned}
& X=K_{1} t^{\frac{2}{3}} \theta^{\frac{\gamma}{2 \gamma-1}}\left(\frac{\theta+1}{2}\right)^{-\frac{2}{3}}\left(\frac{(2-\gamma) \theta+2 \gamma-1}{\gamma+1}\right)^{\frac{5 \gamma^{2}+\gamma-4}{3(2-\gamma)(2 \gamma-1)}} \\
& x=K_{1} t^{\frac{2}{3}} \theta^{\frac{\gamma-1}{2 \gamma-1}}\left(\frac{\theta+1}{2}\right)^{-\frac{2}{3}} \frac{\gamma+\theta}{\gamma+1}\left(\frac{(2-\gamma) \theta+2 \gamma-1}{\gamma+1}\right)^{-\frac{5 \gamma^{2}+\gamma-4}{3(2 \gamma-1)(\gamma+1)}} \\
& \rho=\frac{\gamma+1}{\gamma-1} \rho_{0} \theta^{\frac{1}{2 \gamma-1}}\left(\frac{(2-\gamma) \theta+2 \gamma-1}{\gamma+1}\right)^{\frac{5 \gamma^{2}+\gamma-4}{(2-\gamma)(2 \gamma-1)(\gamma+1)}} \\
& u=\frac{4 K_{1}}{3(\gamma+1)} t^{-\frac{1}{3}} \theta^{\frac{\gamma-1}{2 \gamma-1}}\left(\frac{\theta+1}{2}\right)^{\frac{1}{3}}\left(\frac{(2-\gamma) \theta+2 \gamma-1}{\gamma+1}\right)^{-\frac{5 \gamma^{2}+\gamma-4}{3(2 \gamma-1)(\gamma+1)}} \\
& p=\frac{8 K_{1}^{2}}{9(\gamma+1)} \rho_{0} t^{-\frac{2}{3}}\left(\frac{\theta+1}{2}\right)^{\frac{2}{3}}\left(\frac{(2-\gamma) \theta+2 \gamma-1}{\gamma+1}\right)^{\frac{5 \gamma^{2}+\gamma-4}{3(2-\gamma)(\gamma+1)}}
\end{aligned}
$$

[Fig. 8 about here.]

The constant $K_{1}$, which depends on the energy release $E_{0}$ and the initial fluid density $\rho_{0}$, is given by

$$
K_{1}=1.22904 \sqrt[3]{\frac{E_{0}}{\rho_{0}}} .
$$

In simulations, the field variables are initialized using the exact solution and adopting a finite radius of the explosion such that the steep field profiles can be resolved with enough accuracy with the chosen discretization. The computation proceeds by integrating the flow equations in time following the method described in Section 2. Figure 8 shows snapshots of the pressure profiles computed for an explosion with an energy $E_{0}=5 \times 10^{8} \mathrm{~kg} / \mathrm{s}^{-2}$ corresponding to $111 \mathrm{~kg} / \mathrm{m}^{2}$ of TNT. In this simulation, the blast front is initially located at a radius $\mathcal{R}=22.5 \mathrm{~m}$. The pressure profiles feature two strong discontinuities propagating to the left and to the right with the discontinuous jumps being about three times larger than the pressure at the explosion center. The figure includes a comparison with the exact solution corresponding to the same energy release but at the point when the front has reached a radius $\mathcal{R}=81.2$ m. It may be seen in the comparison that the simulation preserves the shape 
of the pressure profile as it evolves in time.

[Fig. 9 about here.]

Representative temperature and density profiles corresponding to this simulation are shown in Figure 9. As it may be seen in this figure, the accuracy of the numerical solution near the center of the explosion decreases due to the singular character of the solution at the explosion center, which is manifested by the temperature growing unboundedly and the density tending to zero. The numerical solution exhibits a flat density distribution, whereas the temperature peak is finite and clearly unresolved. Refining the solution at the origin only improves the accuracy for a short time in the simulation, as the grid cell at the origin grows substantially faster than its neighboring elements also due to the singularity. However, convergence studies showed that this does not affect the solution far from the origin. In particular, the fluid-plate interaction and the resulting dynamics of the plate remained ostensibly unaffected by the grid resolution at the origin.

[Fig. 10 about here.]

[Fig. 11 about here.]

The computation of the interaction between the blast wave described above and two plates with the density of steel $\left(\rho_{p}=7,800 \mathrm{~kg} / \mathrm{m}^{3}\right)$ and thicknesses $h_{p}=2 \mathrm{~mm}$ and $20 \mathrm{~mm}$ resulted in the plate displacement and pressure histories shown in Figures 11 and 10. As concluded in previous work [12,11], the instantaneous peak pressure on the plate surface is the same regardless of the plate thickness, but the lighter plate accelerates faster away from the blast wave, which relieves the pressure on the surface. This can be clearly seen in 
Figure 10 where the pressure on the $2 \mathrm{~mm}$-thick plate decays significantly faster than the pressure on the $20 \mathrm{~mm}$-thick plate. It should be emphasized, however, that this pressure relief comes at the expense of large displacements of the plate whose motion must be unconstrained for the impulse reduction to be realized. As it can be seen from Figure 11 the required displacements can be significant (on the order of tens of meters) which practically defeats the ambition of exploiting FSI for the purpose of designing blast-protective structures. This statement is confirmed by the unrealistically large velocities reached by the plates (Figure 11)

\section{Conclusions}

A numerical method has been used to simulate the interaction of linear and nonlinear one-dimensional waves propagating in air with the dynamics of a plate hereby represented by a concentrated mass. The interest of this analysis is in evaluating the amount of momentum imparted by the blast to the structure which, owing to the FSI effect, can be substantially reduced. The method has been verified against well-known and recently-derived exact results for blast profiles with uniform and exponential profiles in the limits of either acoustic waves with arbitrary plate mass or arbitrary blast intensity but very heavy or very light plates. More realistic blast profiles initially given by the exact point-source explosion of von Neumann and Sedov have also been considered.

Similarly to what has been found before, the use of lighter plates has the benefit of reducing the transmitted impulse, which potentially can be exploited in structural designs with improved blast resistance, e.g. sandwich plates with 
light front sheets. However, such designs need to take into account the large displacements of the front face sheet required in order for the impulse reduction to take place.

\section{Acknowledgments}

This research was supported by the U.S. Army through the Institute for Soldier Nanotechnologies, under Contract DAAD-19-02-D-0002 with the U.S. Army Research Office. The content does not necessarily reflect the position of the Government, and no official endorsement should be inferred.

\section{References}

[1] G. Taylor, The pressure and impulse of submarine explosion waves on plates, in: G. Batchelor (Ed.), The scientific papers of Sir Geoffrey Ingram Taylor, Vol. III: Aerodynamics and the Mechanics of Projectiles and Explosions, Cambridge University Press, 1963, pp. 287-303.

[2] Z. Xue, J. W. Hutchinson, Preliminary assessment of sandwich plates subject to blast loads, International Journal of Mechanical Sciences 45 (4) (2003) 687 $-705$.

[3] N. Fleck, V. Deshpande, The resistance of clamped sandwich beams to shock loading, Journal of Applied Mechanics, Transactions ASME 71 (3) (2004) 386 -401 .

[4] Z. Xue, J. W. Hutchinson, A comparative study of impulse-resistant metal sandwich plates, International Journal of Impact Engineering 30 (10) (2004) $1283-1305$. 
[5] X. Qiu, V. Deshpande, N. Fleck, Dynamic response of a clamped circular sandwich plate subject to shock loading, Journal of Applied Mechanics, Transactions ASME 71 (5) (2004) 637 - 645.

[6] X. Qiu, V. Deshpande, N. Fleck, Impulsive loading of clamped monolithic and sandwich beams over a central patch, Journal of the Mechanics and Physics of Solids 53 (5) (2005) 1015 - 1046.

[7] J. W. Hutchinson, Z. Xue, Metal sandwich plates optimized for pressure impulses, International Journal of Mechanical Sciences 47 (4-5 SPEC ISS) (2005) $545-569$.

[8] V. Deshpande, N. Fleck, One-dimensional response of sandwich plates to underwater shock loading, Journal of the Mechanics and Physics of Solids 53 (11) (2005) 2347-2383.

[9] T. Rabczuk, J. Y. Kim, E. Samaniego, B. T., Homogenization of sandwich structures, International Journal of Numerical Methods in Engineering 61 (7) (2004) 1009-1027.

[10] Y. Liang, A. V. Spuskanyuk, S. E. Flores, D. R. Hayhurst, H. J. W., R. M. McMeeking, E. A. G., The response of metal sandwitch panels to water blasts, Journal of Applied Mechanics in press.

[11] N. Kambouchev, L. Noels, R. Radovitzky, Fluid-structure interaction effects in the loading of free-standing plates by uniform shocks, Journal of Applied Mechanics.

[12] N. Kambouchev, L. Noels, R. Radovitzky, Compressibility effects in fluidstructure interaction and their implications on the air-blast loading of structures, Journal of Applied Physics.

[13] P. Bulson, Explosive loading of engineering structures, E \& FN Spon, 1997. 
[14] P. Smith, J. Hetherigton, Blast and ballistic loading of structures, Butterworth Heinemann, 1994.

[15] J. von Neumann, The point source solution, in: Collected Works, Vol. 6, Pergamon, 1943, pp. 219-237.

[16] J. von Neumann, R. Richtmyer, A method for the numerical computation of hydrodynamic shocks, Journal of Applied Physics 21 (1950) 232-237.

[17] V. Kuropatenko, On difference methods for the equations of hydrodynamics, in: N. Janenko (Ed.), Difference Methods for Solutions of Problems of Mathematical Physics, Vol. I, American Mathematical Society, 1967, pp. 287303.

[18] D. S. Drumheller, Introduction to Wave Propogation in Nonlinear Fluids and Solids, Cambridge University Press, 1998.

[19] J. Anderson, Fundamentals of Aerodynamics, McGraw-Hill, 2001.

[20] L. Sedov, Similarity and Dimensional Methods in Mechanics, CRC Press, 1993.

[21] G. Taylor, The formation of a blast wave by a very intense explosion, I theorical discussion, Proceeding of Royal Society, Series A 201 (1065) (1950) 159-174.

[22] G. G. Bach, J. H. Lee, Higher-order perturbation solutions for blast waves., AIAA J. 7 (1969) 742.

[23] G. G. Bach, J. H. Lee, An analytical solution for blast waves, AIAA J. 8 (1970) 271. 


\section{List of Figures}

1 Problem of interest: a wave with peak overpressure $p_{s}$ traveling through medium with properties $p_{0}$ and $\rho_{0}$ impinges on a free plate of thickness $h_{p}$ and density $\rho_{p}$. Initial load balance until the shock arrival is provided by the pressure $p_{0}$ applied on the right side of the plate.

2 Verification of the numerical method. Exact pressure reflection coefficients for normal reflection of uniform shocks on a fixed boundary, equation (25), are accurately reproduced for a wide range of shock intensities.

3 Time histories of the overpressure on the surface of the plate for different values of the compressible non-dimensional parameter $\beta_{s}$ and incident shock overpressure $p_{s} / p_{0}=4.49$.

4 Plate impulse time histories for different values of the compressible non-dimensional parameter $\beta_{s}$ and incident shock overpressure $p_{s} / p_{0}=4.49$.

$5 \quad$ Impulse transmission as function of the compressible parameter $\beta_{s}$ for different values of the incident overpressure $p_{s} / p_{0}$.

$6 \quad$ Location of the plate as a function of time for different values of the compressible non-dimensional parameter $\beta_{s}$ and incident overpressure $p_{s} / p_{0}=4.49$.

7 Comparison of numerical results for exponential incident pressure profiles with theory presented in [12]: Transmitted impulse vs. compressible FSI parameter for different blast intensities. Symbols represent numerical results; lines correspond to empirical formula (32).

8 Presure profiles of a one-dimensional point source explosion at different time instants for $E_{0}=5 \times 10^{8} \mathrm{~kg} / \mathrm{s}^{-2}$

9 Representative temperature and density profiles of a one-dimensional point source explosion corresponding to $E_{0}=5 \times 10^{8} \mathrm{~kg} / \mathrm{s}^{-2}$

10 Pressure history $p_{p}$ on a plate initially $32 \mathrm{~m}$ away from explosion center

11 Displacement $\xi$ and velocity $\dot{\xi}$ history of a plate initially $32 \mathrm{~m}$ away from explosion center 


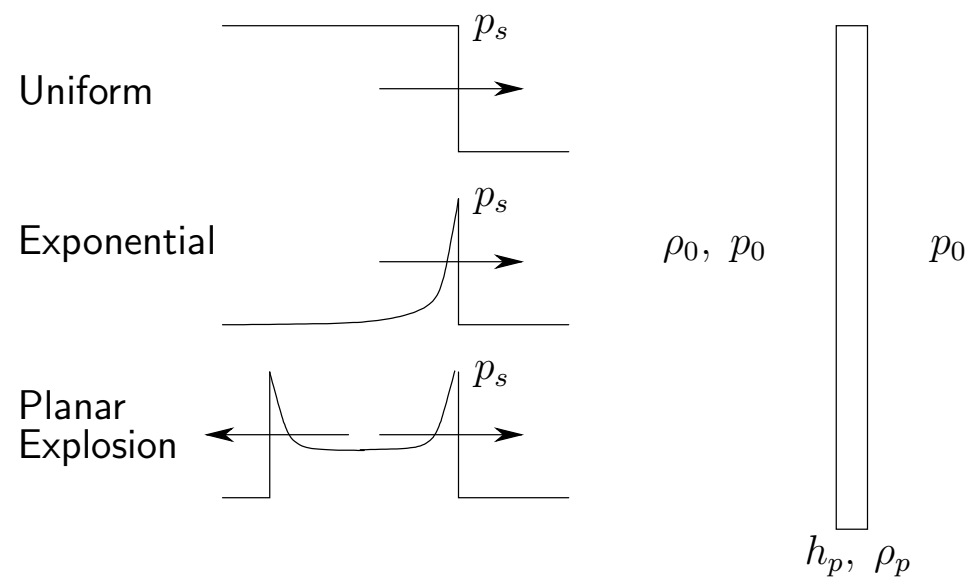

Fig. 1. Problem of interest: a wave with peak overpressure $p_{s}$ traveling through medium with properties $p_{0}$ and $\rho_{0}$ impinges on a free plate of thickness $h_{p}$ and density $\rho_{p}$. Initial load balance until the shock arrival is provided by the pressure $p_{0}$ applied on the right side of the plate. 


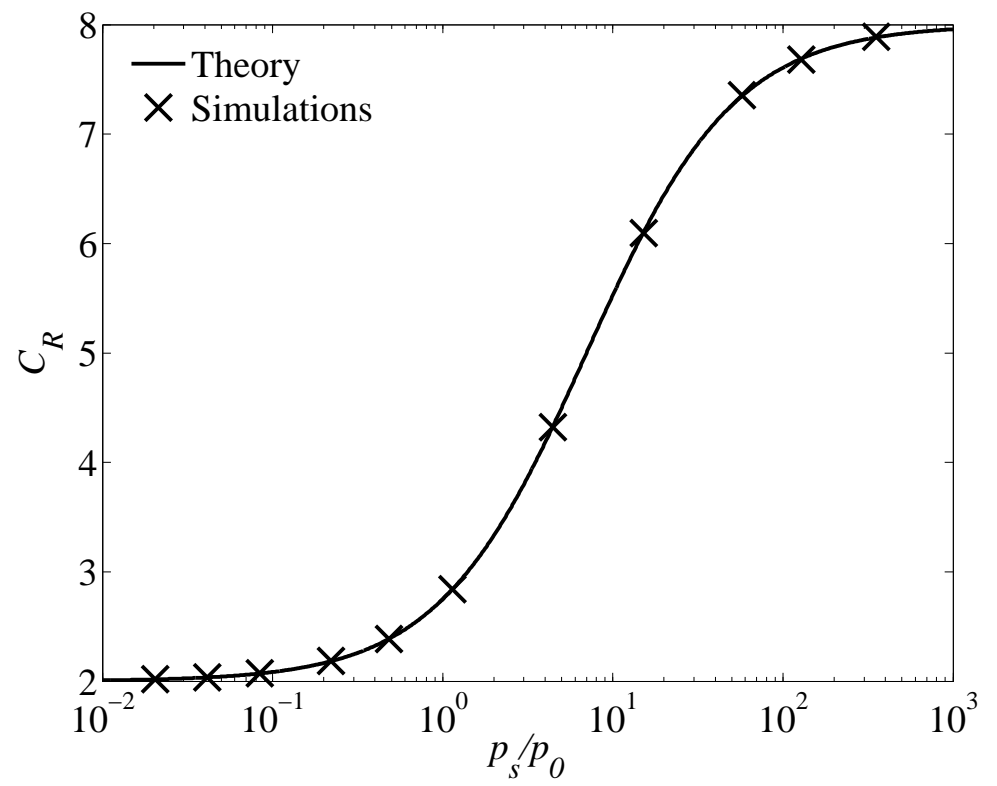

Fig. 2. Verification of the numerical method. Exact pressure reflection coefficients for normal reflection of uniform shocks on a fixed boundary, equation (25), are accurately reproduced for a wide range of shock intensities. 


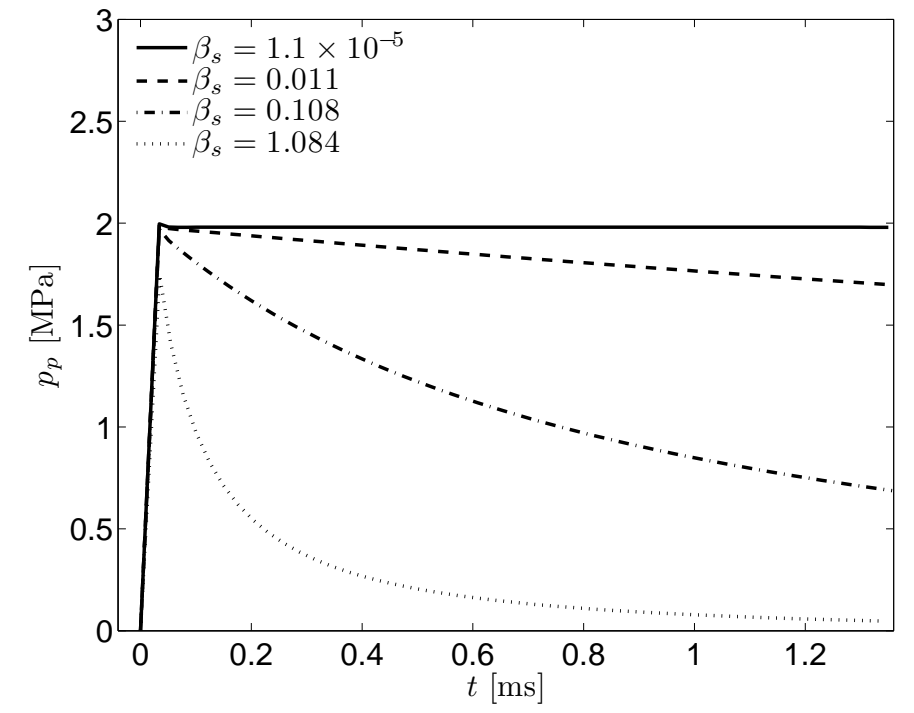

Fig. 3. Time histories of the overpressure on the surface of the plate for different values of the compressible non-dimensional parameter $\beta_{s}$ and incident shock overpressure $p_{s} / p_{0}=4.49$. 


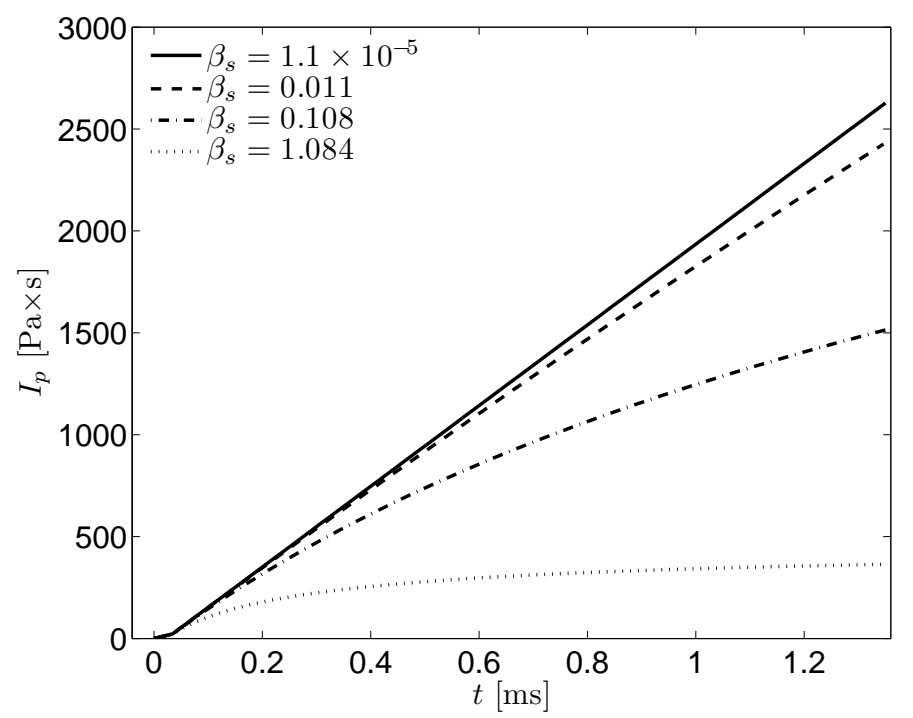

Fig. 4. Plate impulse time histories for different values of the compressible non-dimensional parameter $\beta_{s}$ and incident shock overpressure $p_{s} / p_{0}=4.49$. 


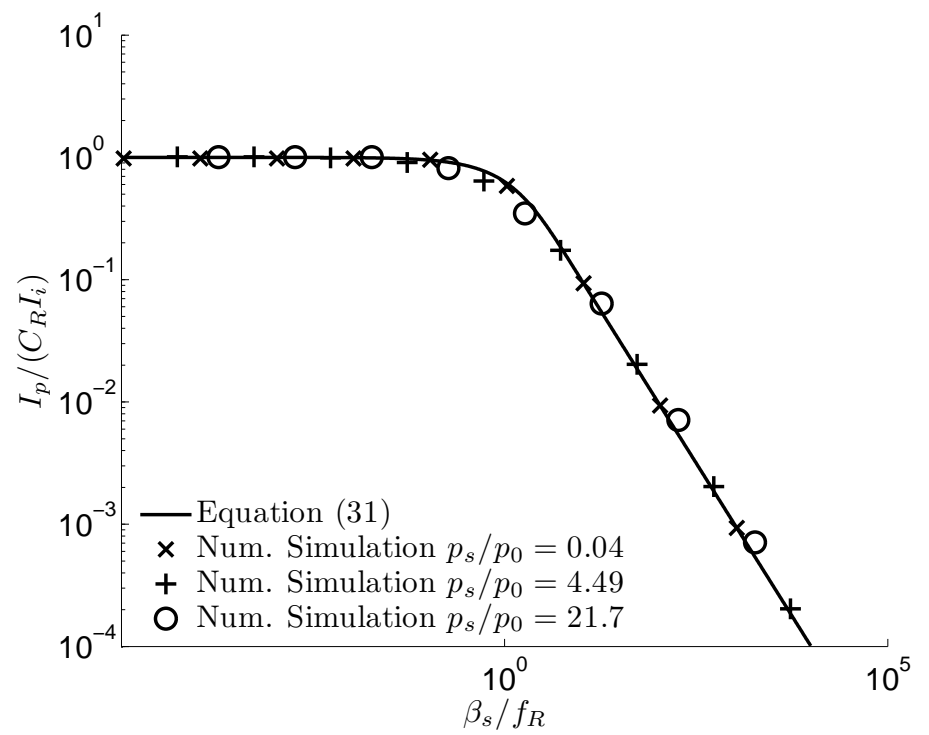

Fig. 5. Impulse transmission as function of the compressible parameter $\beta_{s}$ for different values of the incident overpressure $p_{s} / p_{0}$. 


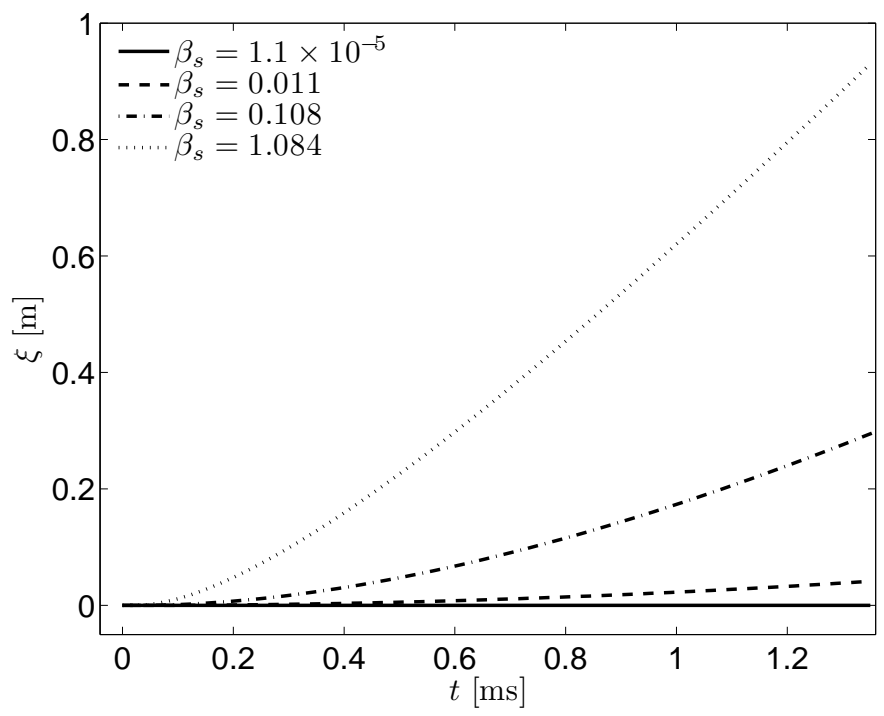

Fig. 6. Location of the plate as a function of time for different values of the compressible non-dimensional parameter $\beta_{s}$ and incident overpressure $p_{s} / p_{0}=4.49$. 


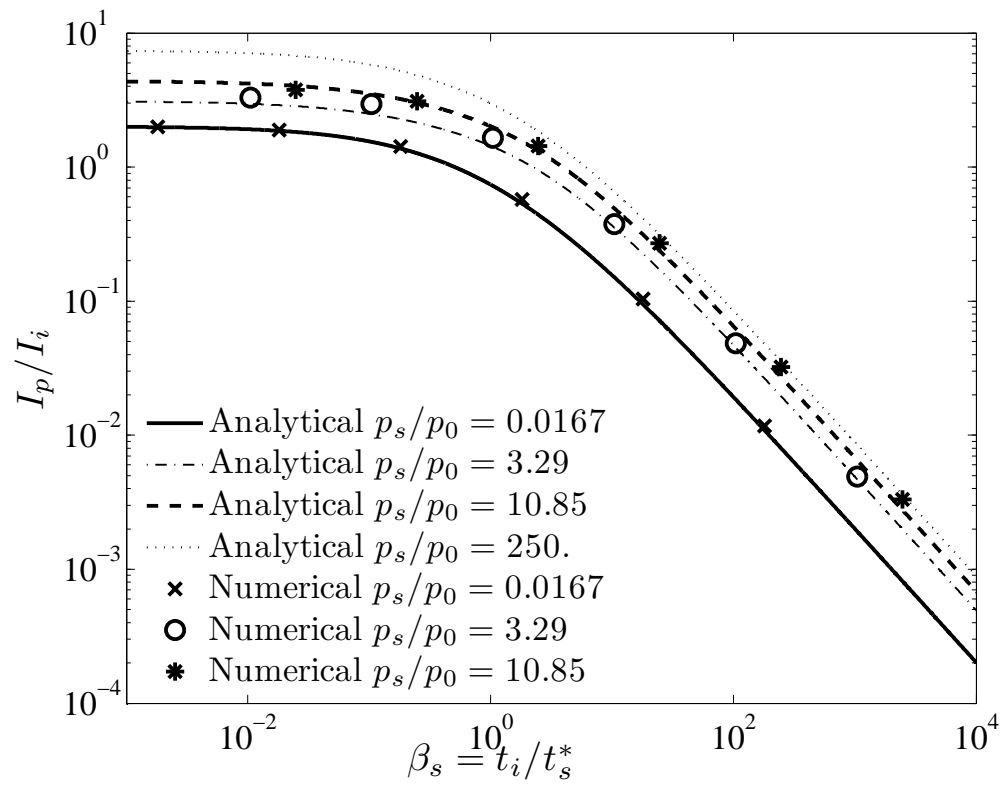

Fig. 7. Comparison of numerical results for exponential incident pressure profiles with theory presented in [12]: Transmitted impulse vs. compressible FSI parameter for different blast intensities. Symbols represent numerical results; lines correspond to empirical formula (32). 


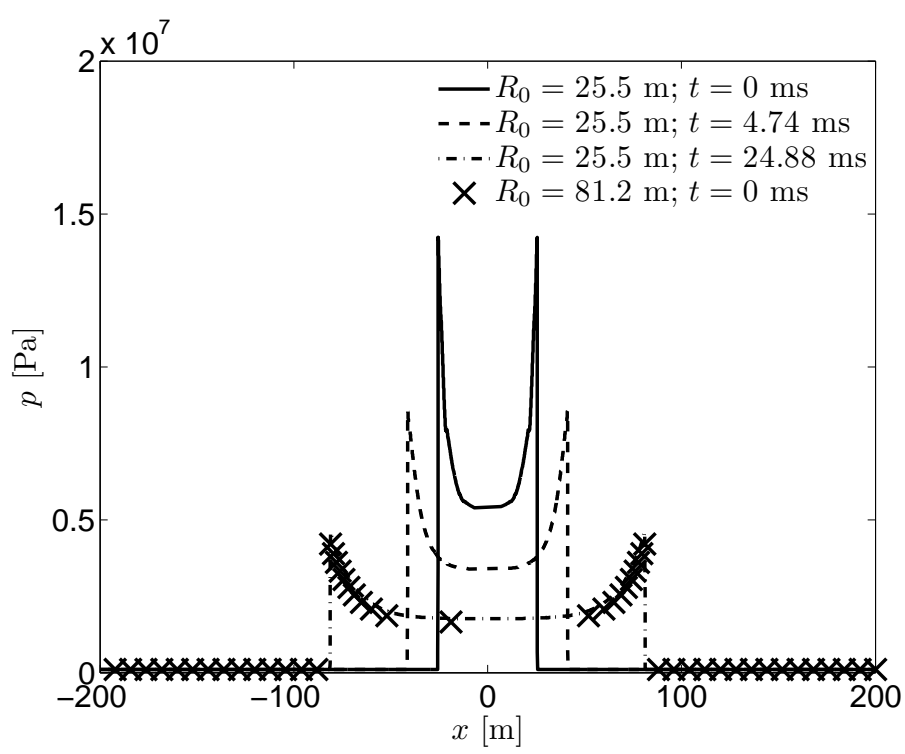

Fig. 8. Presure profiles of a one-dimensional point source explosion at different time instants for $E_{0}=5 \times 10^{8} \mathrm{~kg} / \mathrm{s}^{-2}$ 


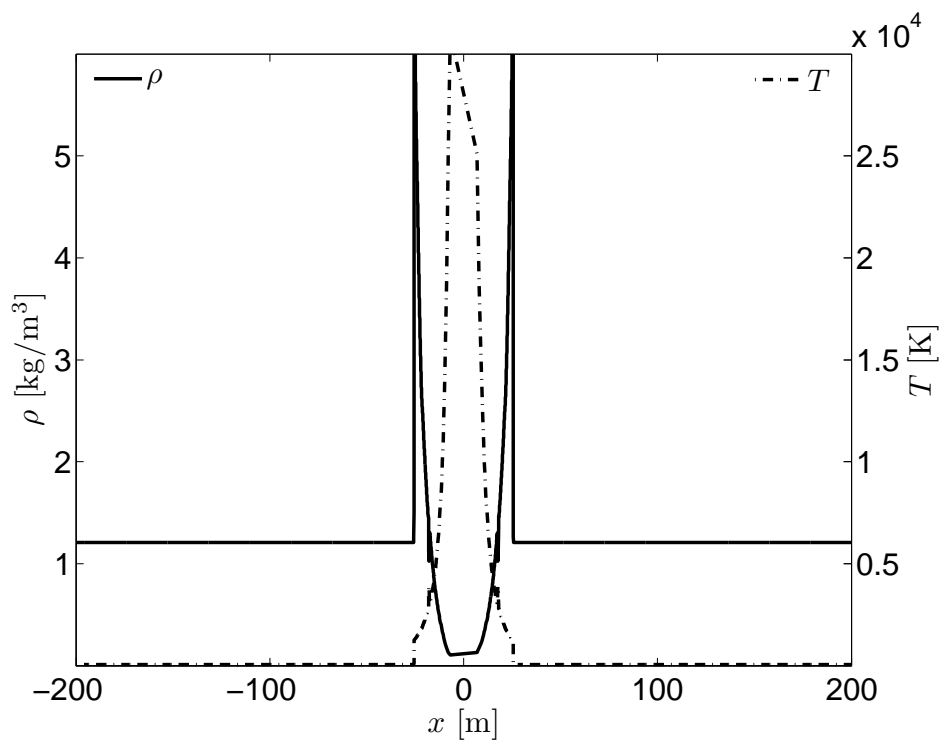

Fig. 9. Representative temperature and density profiles of a one-dimensional point source explosion corresponding to $E_{0}=5 \times 10^{8} \mathrm{~kg} / \mathrm{s}^{-2}$ 


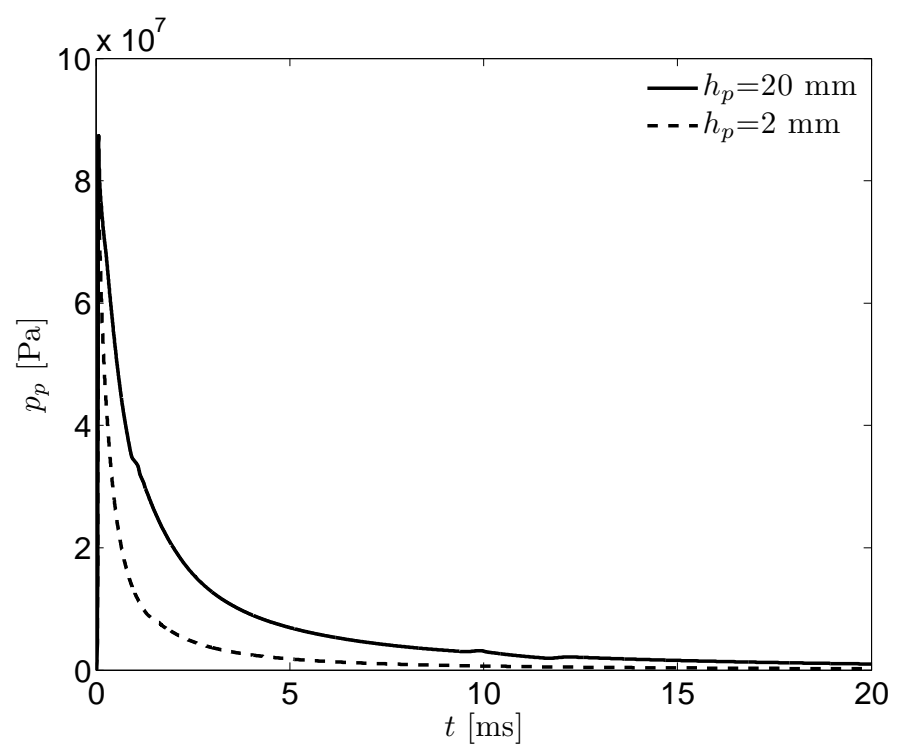

Fig. 10. Pressure history $p_{p}$ on a plate initially $32 \mathrm{~m}$ away from explosion center 


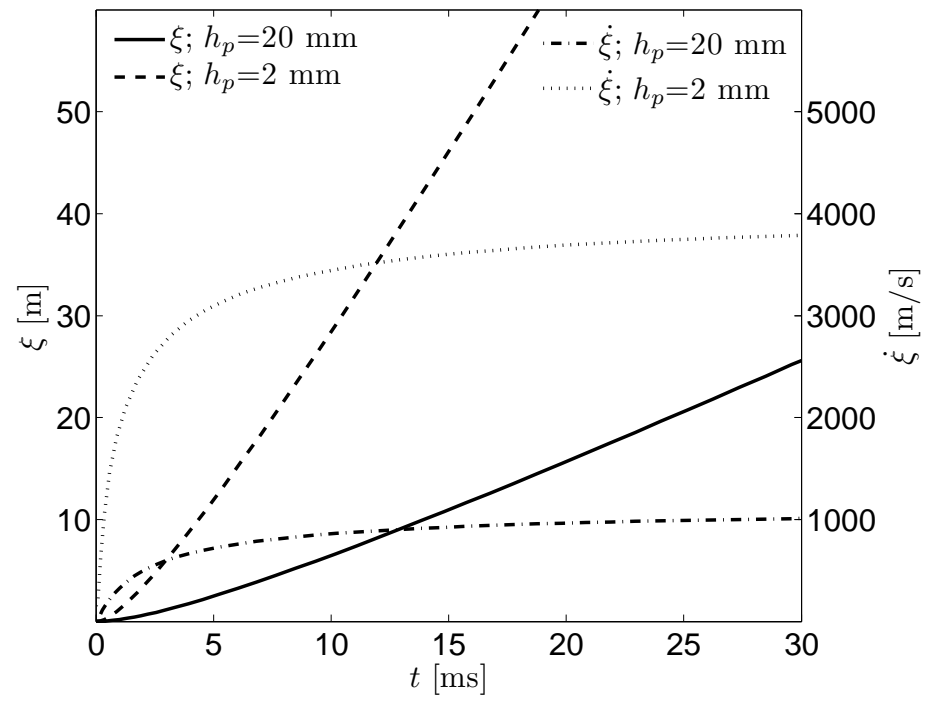

Fig. 11. Displacement $\xi$ and velocity $\dot{\xi}$ history of a plate initially $32 \mathrm{~m}$ away from explosion center 